\title{
Chaotic Vibrations of the Duffing System with Fractional Damping
}

\author{
$\underline{\text { Grzegorz Litak }^{\dagger}}$, Arkadiusz Syta $^{\dagger}$, Stefano Lenci $^{\ddagger}$, Michael Scheffler* \\ ${ }^{\dagger}$ Faculty of Mechanical Engineering, Lublin University of Technology \\ PL-20-618 Lublin, Poland \\ $\{$ g.litak|a.syta\}@pollub.pl \\ $\ddagger$ Department of Civil and Buildings Engineering, and Architecture \\ Polytechnic University of Marche, Via Brecce Bianche, I-60131 Ancona, Italy \\ lenci@univpm.it
}
* Institut für Festkörpermechanik Professur für Dynamik und Mechanismentechnik Technische Universität Dresden D-01062 Dresden, Germany
michael.scheffler@tu-dresden.de

\begin{abstract}
We examine the Duffing system with a fractional damping term. Instead of the standard Lyapunov exponent calculations, we propose a statistical 0-1 test for chaos detection. For the investigated deterministic model we are able to show a transition from chaotic to regular motion increasing the fractional power of the damping term derivative. The results have been confirmed by a bifurcation diagram.
\end{abstract}

We start from the Duffing equation with an additional fractional damping term

$$
\frac{\mathrm{d}^{2} x}{\mathrm{~d} t^{2}}+\alpha \frac{\mathrm{d}^{q} x}{\mathrm{~d} t^{q}}-x+x^{3}=\delta \cos (\omega t)
$$

where $\frac{\mathrm{d}^{q} x}{\mathrm{~d} t^{q}}$ is the Grünwald-Letnikov fractional derivative $[1,2]$

$$
\frac{\mathrm{d}^{q} x}{\mathrm{~d} t^{q}}={ }_{a} D_{t}^{q} x(t)=\lim _{h \rightarrow 0} \frac{1}{h^{q}} \sum_{j=0}^{\left[\frac{t-a}{h}\right]}(-1)^{j}\left(\begin{array}{l}
q \\
j
\end{array}\right) x(t-j h),
$$

where binomial coefficients can be expressed by the Euler's Gamma function

$$
\left(\begin{array}{l}
q \\
j
\end{array}\right)=\frac{q !}{j !(q-j) !}=\frac{\Gamma(q+1)}{\Gamma(j+1) \Gamma(q-j+1)},
$$

where $[y]$ means the integer part of $y$, and where $a$ is an arbitrary number lesser than $t$.

The dynamical response of the system is studied by changing the fractional power $q$ and by keeping fixed initial conditions $x_{0}=0.21$ and $\dot{x}_{0}=0.31$. The time histories are plotted in Fig. 1 (systems parameters $\alpha=0.15, \delta=0.3, \omega=1.0, a=0$ ). Figures 1(a) and 1(b) show initial non-periodic transients and final regular motion, while the response in Fig. 1(c) is chaotic.

To clarify the transition from Fig. 1(a) to Fig. 1(c) the system response is examined more systematically by means of bifurcation diagram (Fig. 2(a)) and by the $0-1$ test [3] (Fig. 2(b)). The 
(a)

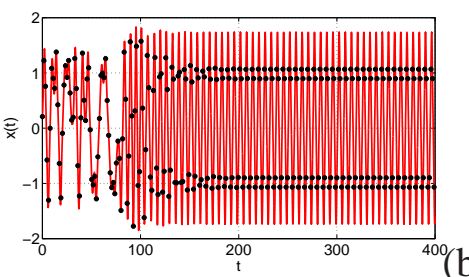

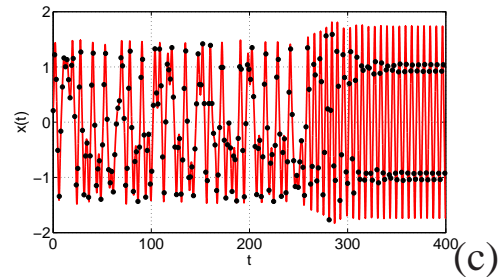

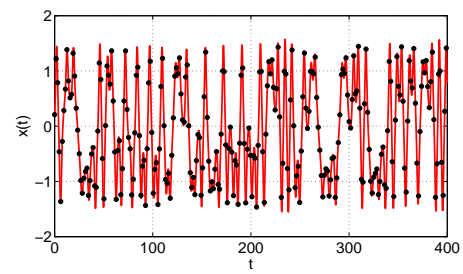

Figure 1: Time series of displacement $x$ (a)-(c) for different derivative orders $q$ of the damping term, $q=0.6,0.7,0.8$, respectively. Isolated black points indicate the sampling points with the sampling period $\Delta t=T_{0} / 4$ (the excitation period is $T_{0}=2 \pi / \omega$ ).

0-1 test combines both spectral and statistical properties of the system and can distinguish the dynamics of the system by value $K \in[0,1]$. Applying the test we used a modified Poincaré section (see black points in Fig. 1) to record 4 points per period $T=2 \pi / \omega$ which led us both to lower computational effort and to preserve the characteristics of system response.

For increasing values of $q \in(0,2)$ one can see the alternating regions of chaotic and regular motions. Comparing Figs. 2(a) and 2(b) one can see the agreement in the results of examined methods. In summary, the $0-1$ method can be used instead of other nonlinear method such the maximal Lyapunov exponent which is more difficult to estimate in the system with memory.

(a)

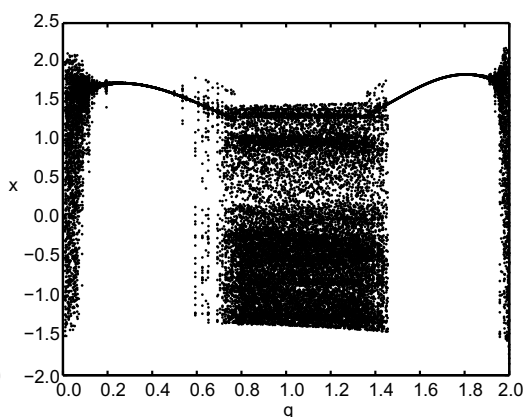

(b)

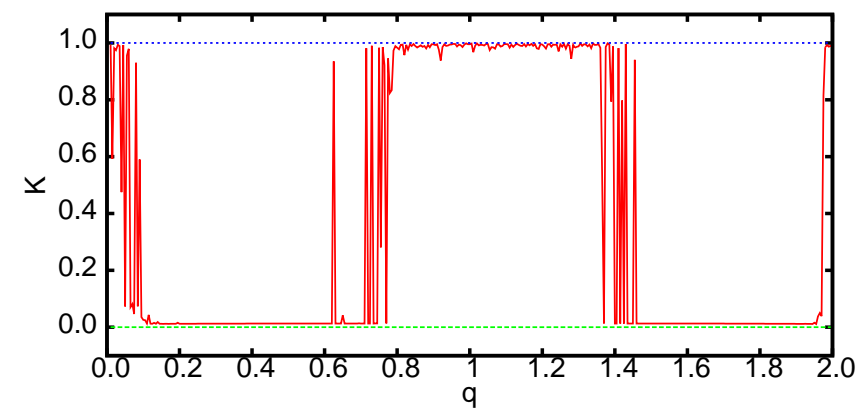

Figure 2: The bifurcation diagram of the $x$ coordinate (a) and the $K$ parameter of the $0-1$ test (b), taking 0 value for regular and 1 for chaotic solutions, versus the fractional order $q \in(0,2)$.

Acknowledgement. The research leading to these results has received funding from the European Union Seventh Framework Programme (FP7/2007-2013), FP7 - REGPOT - 2009 - 1, under grant agreement No:245479.

\section{References}

[1] Y.A. Rossikhin and M.V. Shitikova, Application of fractional calculus for dynamic problems of solid mechanics: Novel trends and recent results. Appl. Mech. Rev. 63:010801, 2010 .

[2] I. Petras, Fractional-Order Nonlinear Systems: Modeling, Analysis and Simulation, Springer 2011.

[3] G.A. Gottwald and I. Melbourne, On the implementation of the 0-1 test for chaos. SIAM J. Appl. Dyn. Syst. 8:129-145, 2009. 